\title{
Peptide Micelles for Anti-cancer Drug Delivery in an Intracranial Glioblastoma Animal Model
}

\author{
Na Yi and Minhyung Lee* \\ BK21 Plus Future Biopharmaceutical Human Resources Training and Research Team, Department of Bioengineering, \\ College of Engineering, Hanyang University, Seoul 133-791, Korea. ${ }^{*}$ E-mail: minhyung@hanyang.ac.kr \\ Received May 7, 2014, Accepted June 26, 2014
}

\begin{abstract}
Bis-chloroethylnitrosourea (BCNU) is currently used as an anti-cancer drug for glioblastoma therapy. In this study, BCNU was loaded into the hydrophobic cores of R3V6 amphiphilic peptide micelles for efficient delivery into brain tumors. The scanning electron microscope (SEM) study showed that the BCNU-loaded R3V6 peptide micelles (R3V6-BCNU) formed spherical micelles. MTT assay showed that R3V6-BCNU more efficiently induced cell death in $\mathrm{C} 6$ glioblastoma cells than did BCNU. In the Annexin V assay, R3V6-BCNU more efficiently induced apoptosis than did BCNU alone. Furthermore, the results showed that R3V6 was not toxic to cells. The positive charges of the R3V6 peptide micelles may facilitate the interaction between R3V6$\mathrm{BCNU}$ and the cellular membrane, resulting in an increase in cellular uptake of BCNU. In vivo evaluation with an intracranial glioblastoma rat model showed that R3V6-BCNU more effectively reduced tumor size than BCNU alone. The results suggest that R3V6 peptide micelles may be an efficient carrier of BCNU for glioblastoma therapy.
\end{abstract}

Key Words : Glioblastoma, BCNU, Drug delivery, Peptide micelle, Animal model

\section{Introduction}

Glioblastoma multiforme (GBM) is the most malignant form of brain tumor. ${ }^{1,2}$ The treatments for glioblastoma include surgery, chemotherapy, and radiotherapy. However, the surgical removal of tumors from the brain is not easy, since an aggressive dissection may cause severe side-effects in brain function. GBM rarely metastasizes to other organs; however, the internal migration of the tumor within the brain is a common event. Due to this migration, GBM recurrence after surgery is common. Therefore, the combination of chemotherapy and radiotherapy with surgery is inevitable for the treatment of GBM. The average lifespan of GBM patients is less than one year.

GBM has hypoxic regions in its core, and hypoxia has been shown to induce cell death in the cores of the tumors. ${ }^{2}$ However, hypoxia has a negative effect on cancer therapy because the lack of blood capillaries limits the free diffusion of anticancer drug into the core of the tumor. Furthermore, tumor stem cells in solid tumors are usually found within hypoxic regions. ${ }^{3}$ Therefore, hypoxia may induce drug resistance in solid tumors. ${ }^{4,5}$ These findings suggest that antitumor drugs must be efficiently delivered to tumors in order to achieve successful anti-cancer effects.

Bis-chloroethylnitrosourea (BCNU, carmustine) is a widely used drug for glioblastoma. The high toxicity of BCNU induces several significant side-effects. ${ }^{6}$ For the treatment of GBM, a wafer infused with BCNU (Gliadel) is surgically implanted under the skull. ${ }^{7}$ The BCNU released from the wafer can inhibit the recurrence of glioblastoma. However, the application of Gliadel requires a highly invasive surgical procedure. Injectable systems with BCNU-loaded nanoparticles may be useful for the delivery of BCNU to glioblastoma.

R3V6 is an amphiphilic peptide that forms micelles in aqueous solution (Fig. 1). It was previously shown that hydrophobic drugs such as dexamethasone, curcumin, and BCNU could be loaded within the cores of R3V6 peptide micelles. ${ }^{8,9}$ In vitro studies showed that BCNU-loaded R3V6 (R3V6-BCNU) more efficiently delivered siRNA or plasmid DNA (pDNA) than did empty R3V6 micelles. ${ }^{10}$ Furthermore, the R3V6-BCNU/pDNA complex were more toxic to the cancer cells than was the mixture of BCNU and pDNA. ${ }^{10}$

In the current study, R3V6-BCNU was evaluated as a delivery carrier of BCNU without pDNA or siRNA. The drug delivery efficiency of R3V6-BCNU was evaluated in vitro. In addition, its in vivo anti-tumor efficacy was evaluated in an intracranial glioblastoma rat model. The results suggest that R3V6 peptide micelles are useful for the delivery of $\mathrm{BCNU}$ for the treatment of glioblastoma.

\section{Experimental}

Synthesis of R3V6 Peptides. The R3V6 peptides were synthesized chemically by Peptron (Daejeon, Korea). The synthesis of R3V6 was confirmed by mass spectrometry. The synthesized R3V6 peptides were purified by HPLC using a C8 reverse phase column. The purity of the R3V6 peptide was confirmed by HPLC.

Preparation of R3V6-BCNU. BCNU was dissolved in water at $4 \mathrm{mg} / \mathrm{mL}$ (Sigma, St. Louis, MO, USA). The BCNU solution was mixed with the R3V6 solution. After sonication, the mixed solution was incubated at room temperature for 30 min. The weight ratio between R3V6 and BCNU was fixed at 1:0.6 (R3V6:BCNU) based on a previous report. ${ }^{11}$ 


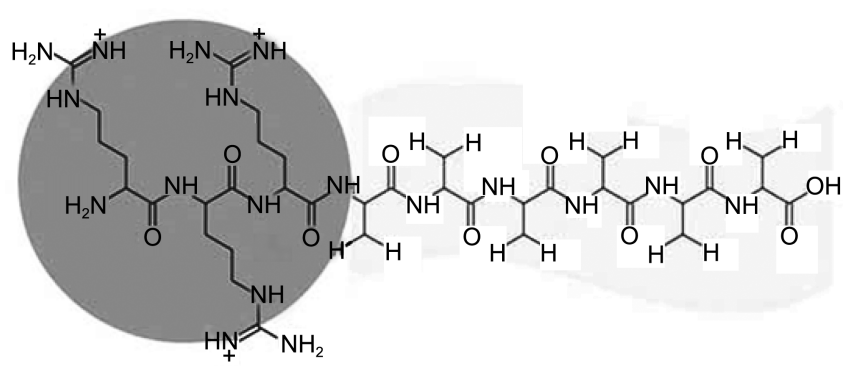

Figure 1. The structure of the R3V6 peptide. The hydrophilic region is marked with a gray circle.

Fluorescent Dye Quenching Assay. Dye quenching assays were performed according to the previously described method. ${ }^{12} \mathrm{R} 3 \mathrm{~V} 6$ was mixed with $10 \mu \mathrm{g}$ of the hydrophobic fluorescent dye, 5-dodecanoylaminofluorescein (DAF), or the hydrophilic fluorescent dye, Oregon green. The weight ratios between R3V6 and the dyes were 0.5:1, 1:1, 1.5:1, $2: 1$, and $5: 1$. Fluorescence was measured using a fluorometer (DAF: excitation, $485 \mathrm{~nm}$ and emission, $538 \mathrm{~nm}$; Oregon green: excitation, $496 \mathrm{~nm}$ and emission, $524 \mathrm{~nm}$ ).

Scanning Electron Microscopy (SEM) Image. R3V6$\mathrm{BCNU}$ was prepared at a 1:1.6 weight ratio (R3V6:BCNU) based on the methods of a previous report. ${ }^{11} \mathrm{R} 3 \mathrm{~V} 6$ or R3V6$\mathrm{BCNU}$ in water was mounted on carbon tapes at room temperature and dried overnight. The dried micelles were coated with silver under vacuum, and their shapes and sizes were investigated by SEM (NANO SEM 450, FEI, Hillsboro, OR, USA).

Cytotoxicity Assay. The MTT assay was performed to evaluate the cytotoxicity of R3V6 or R3V6-BCNU. The C6 glioblastoma cells were seeded at a density of $1.5 \times 10^{4} \mathrm{cells} /$ well in 24-well plates and incubated for $24 \mathrm{~h}$ at $37^{\circ} \mathrm{C}$ in a $5 \% \mathrm{CO}_{2}$ incubator. R3V6-BCNU was prepared at a 1:1.6 weight ratio (R3V6:BCNU). R3V6-BCNU, BCNU only, or R3V6 only were added to cells. The amount of R3V6 or $\mathrm{BCNU}$ was fixed at $8 \mu \mathrm{g}$. The culture media were replaced after $4 \mathrm{~h}$ of incubation. The cells were incubated for an additional $44 \mathrm{~h}$ at $37^{\circ} \mathrm{C}$ in a $5 \% \mathrm{CO}_{2}$ incubator. MTT [3(4,5-dimethylthiazol-2-yl)-2,5-diphenyltetrazolium bromide] in PBS was added to the cells, and the cells were incubated for an additional $4 \mathrm{~h}$ at $37{ }^{\circ} \mathrm{C}$ in a $5 \% \mathrm{CO}_{2}$ incubator. The MTT-containing medium was then removed, and dimethyl sulfoxide (DMSO) was added to dissolve the formazan crystals formed by the live cells. Cell viability was evaluated by measuring the absorbance at $570 \mathrm{~nm}$. Cell viability (\%) was calculated according to the following equation: Cell viability $(\%)=\left(\mathrm{OD}_{570 \mathrm{~nm} \text { (sample) }} / \mathrm{OD}_{570 \mathrm{~nm}(\text { (ontrol) })} \times 100\right.$, where OD570 $\mathrm{nm}$ (sample) indicates the wells treated with the micelles, and OD570 $\mathrm{nm}$ (Control) represents the wells treated with PBS.

Flow Cytometry Analysis. The C6 cells were seeded at a density of $5.0 \times 10^{5}$ cells/well in six-well plates and incubated for 24 h. R3V6-BCNU, BCNU only, or R3V6 only was added to the cells as described above. After $20 \mathrm{hrs}$, the cells were washed, suspended with PBS, and then centrifuged at
$1,200 \mathrm{rpm}$ for $5 \mathrm{~min}$. The cells were then resuspended in FACS buffer and centrifuged at 1,200 rpm for $5 \mathrm{~min}$. The cells were analyzed by flow cytometry to evaluate the apoptosis level (BD FACS Calibur TM, BD Bioscience Immunocytometry Systems, San Jose, CA).

In vivo Experiments in the Intracranial Tumor Rat Model. An intracranial tumor rat model was developed with seven-week-old male Sprague Dawley rats. Intracerebral tumors were generated by stereotactic injections of C6 cells. The rats were anesthetized, and each skull was exposed in order to bore a 2 -mm-diameter hole. The monitoring points 2-mm lateral to bregma were carefully drilled with a 26gauge Hamilton micro-syringe (80330; Hamilton, Reno, NV, USA) using a gentle saline $(0.89 \% \mathrm{NaCl})$ drip in order to avoid dura disruption (coordinates to bregma: anteroposterior, $0 \mathrm{~mm}$; lateral, $2.0 \mathrm{~mm}$; ventral, $4.0 \mathrm{~mm}$ ). After each hole was made, $1 \times 10^{5} \mathrm{C} 6$ cells $/ 10 \mu \mathrm{L}$ saline were injected into the cerebral cortex. The injections were performed by first rapidly injecting $1 \mu \mathrm{L}$ of cells and then slowly injecting $9 \mu \mathrm{L}$ of cells (at a speed of $0.9 \mu \mathrm{L} / \mathrm{min}$ ). One week after tumor implantation, the rats were randomly allocated into four groups (cancer control, R3V6 only, BCNU only, R3V6$\mathrm{BCNU})$ of five rats. A total volume of $10 \mu \mathrm{L}$ of sample $(8 \mu \mathrm{g}$ $\mathrm{BCNU}$ only, $5 \mu \mathrm{g}$ R3V6 only, $13 \mu \mathrm{g}$ R3V6-BCNU, or saline) was stereotaxically injected into the site of the tumor cell implantation. Two weeks after tumor implantation, the rats were sacrificed. The brains were harvested and fixed with $4 \%$ paraformaldehyde solution.

Nissl Staining. The fixed brain samples were embedded in paraffin and cut into sections ( $5 \mu \mathrm{m}$ thick), and Nissl staining was performed to determine the histopathological features of the brains. ${ }^{13}$ After deparaffinization, the brain section slides were incubated in cresyl violet $(0.1 \%$, Sigma, St. Louis, MO, USA), treated with destaining solution $(70 \%$ ethanol, $10 \%$ acetic acid), dehydrated (100\% ethanol and xylene), and coverslipped with mounting solution. The total sizes of the brains and the tumor volumes were measured using Image J 1.42 software (National Institutes of Health, Bethesda, MD, USA).

\section{Results}

Physical Characterization of R3V6 Micelles Loaded with Hydrophobic Drugs. The loading of hydrophobic drug into the R3V6 micelles was confirmed by a dye quenching assay using a hydrophobic dye, DAF. As a control, the hydrophilic dye Oregon green was mixed with the R3V6 peptide micelles. The results showed that the fluorescence of DAF was quenched, suggesting that the hydrophobic DAF was loaded into the core of the micelles (Fig. 2). However, the Oregon green fluorescence was not significantly decreased, suggesting that it was not loaded into the R3V6 peptide micelles (Fig. 2). These results indicate that the hydrophobic dye was selectively loaded into the core of the R3V6 micelles.

The morphology of the drug-loaded R3V6 micelles was confirmed by SEM. The weight ratio of R3V6 and BCNU 


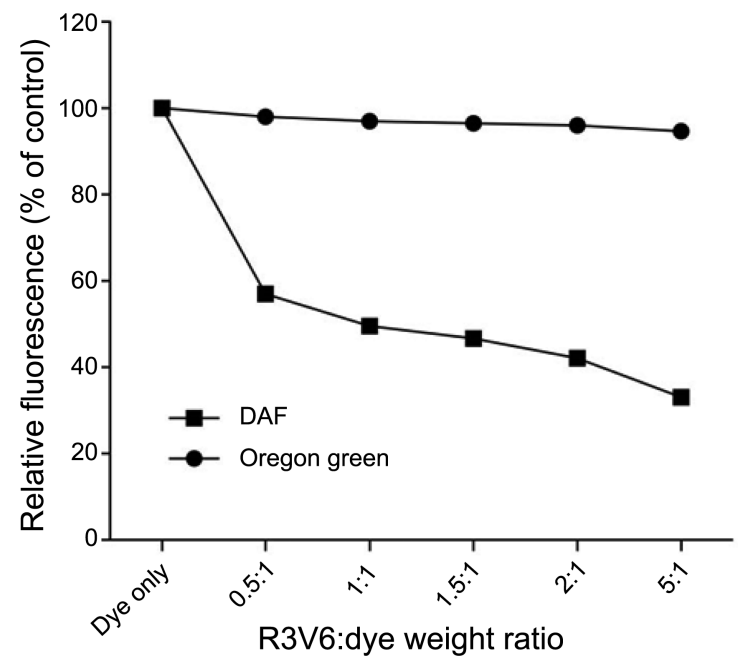

Figure 2. Dye quenching assays. The R3V6 peptides were mixed with $10 \mu \mathrm{g}$ of the hydrophobic fluorescence dye 5-dodecanoylaminofluorescein (DAF) or the hydrophilic fluorescence dye Oregon Green. Fluorescence was measured using a fluorometer.

was fixed at 1:1.6 based on a previous report. ${ }^{11} \mathrm{R} 3 \mathrm{~V} 6$ only or R3V6-BCNU was loaded on a carbon tape and observed by SEM. The results showed that the micelles formed beadlike structures with both R3V6 only and R3V6-BCNU (Fig. 3).

In vitro Anti-cancer Activity of R3V6-BCNU. Anticancer activity of R3V6-BCNU was measured by MTT assays. The ratio between $\mathrm{R} 3 \mathrm{~V} 6$ and $\mathrm{BCNU}$ was optimized.
As a result, R3V6-BCNU at a ratio of 1:1.6 (R3V6:BCNU) showed the highest toxicity to the $\mathrm{C} 6$ glioblastoma cells (Fig. 4(a)). In addition, the cytotoxicity of R3V6-BCNU was compared with R3V6 only and BCNU only The results showed that R3V6-BCNU had the strongest anti-cancer effect (Fig. 4(b)). The same amount of BCNU showed a weaker anti-cancer effect than R3V6-BCNU. In addition, R3V6 only did not reduce cell viability. This suggests that the R3V6 micelles themselves were not toxic to cells. Taken together, the results indicate that R3V6 has a stronger anticancer effect than BCNU only. This suggests that R3V6 may increase the $\mathrm{BCNU}$ delivery efficiency.

The anti-cancer effect of R3V6-BCNU was confirmed by the apoptosis assay with Annexin- $\mathrm{V}$. The Annexin- $\mathrm{V}$ assay showed that R3V6-BCNU induced apoptosis much more efficiently than BCNU only. The ratio of apoptotic cells in the BCNU-only group was $23 \%$, while the ratio in the R3V6-BCNU group was $68.20 \%$ (Fig. 4(c)).

In vivo Evaluation of $\mathrm{R3V6}-\mathrm{BCNU}$ in the Intracranial Glioblastoma Animal Model. The intracranial glioblastoma model was produced by the injection of $\mathrm{C} 6$ cells into the brains of rats using stereotaxic equipment. One week after the $\mathrm{C} 6$ injection, R3V6-BCNU was locally injected into the rat brain using stereotaxic equipment. One week later, the rats were sacrificed, and the brains were harvested for analysis. The results showed that the injection of BCNU only more effectively reduced tumor size compared to injection with the control or R3V6 only (Fig. 5). However,
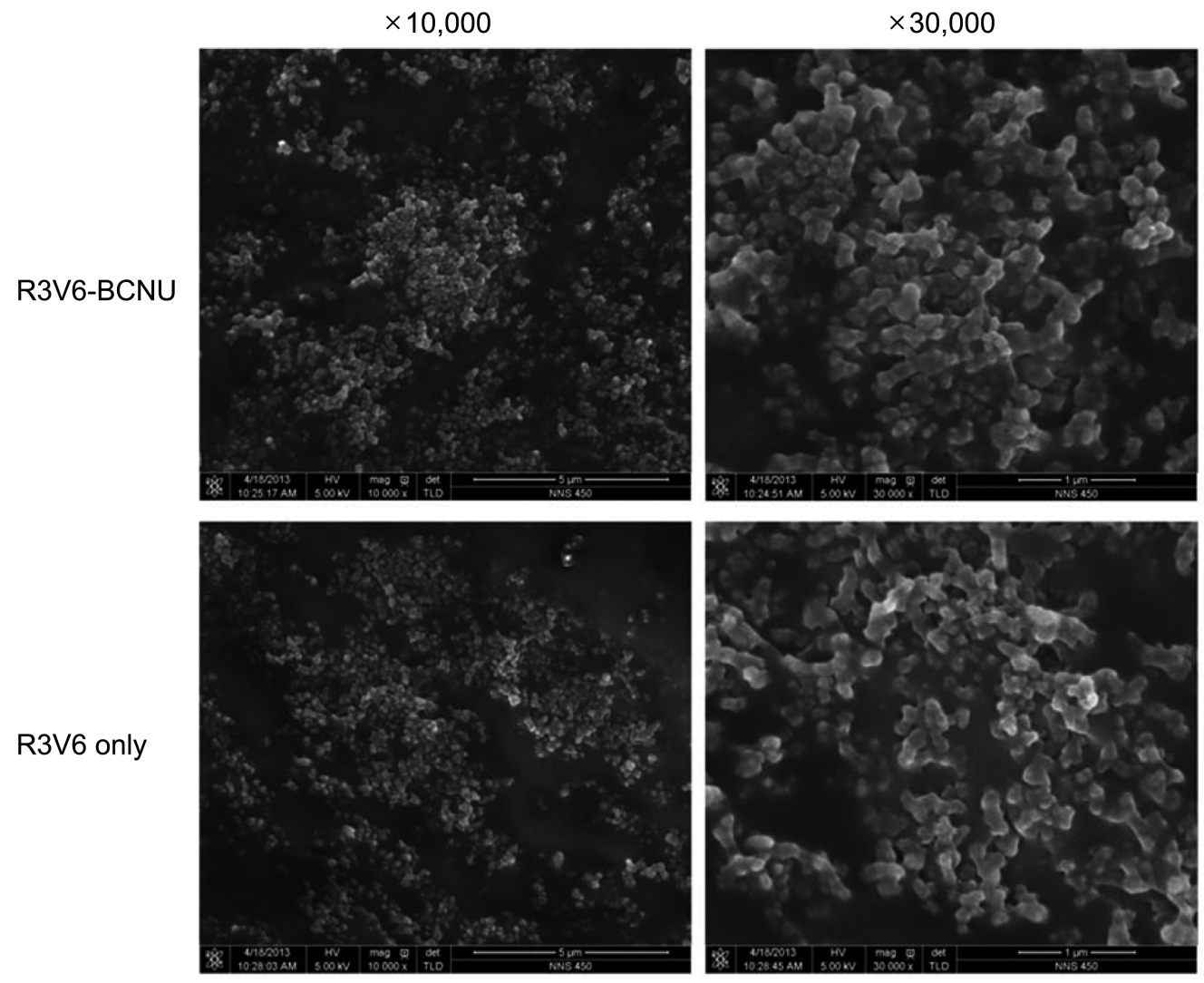

Figure 3. Scanning electron microscopy analysis of R3V6-BCNU. The morphology of R3V6-BCNU and R3V6 only was observed by scanning electron microscopy. 

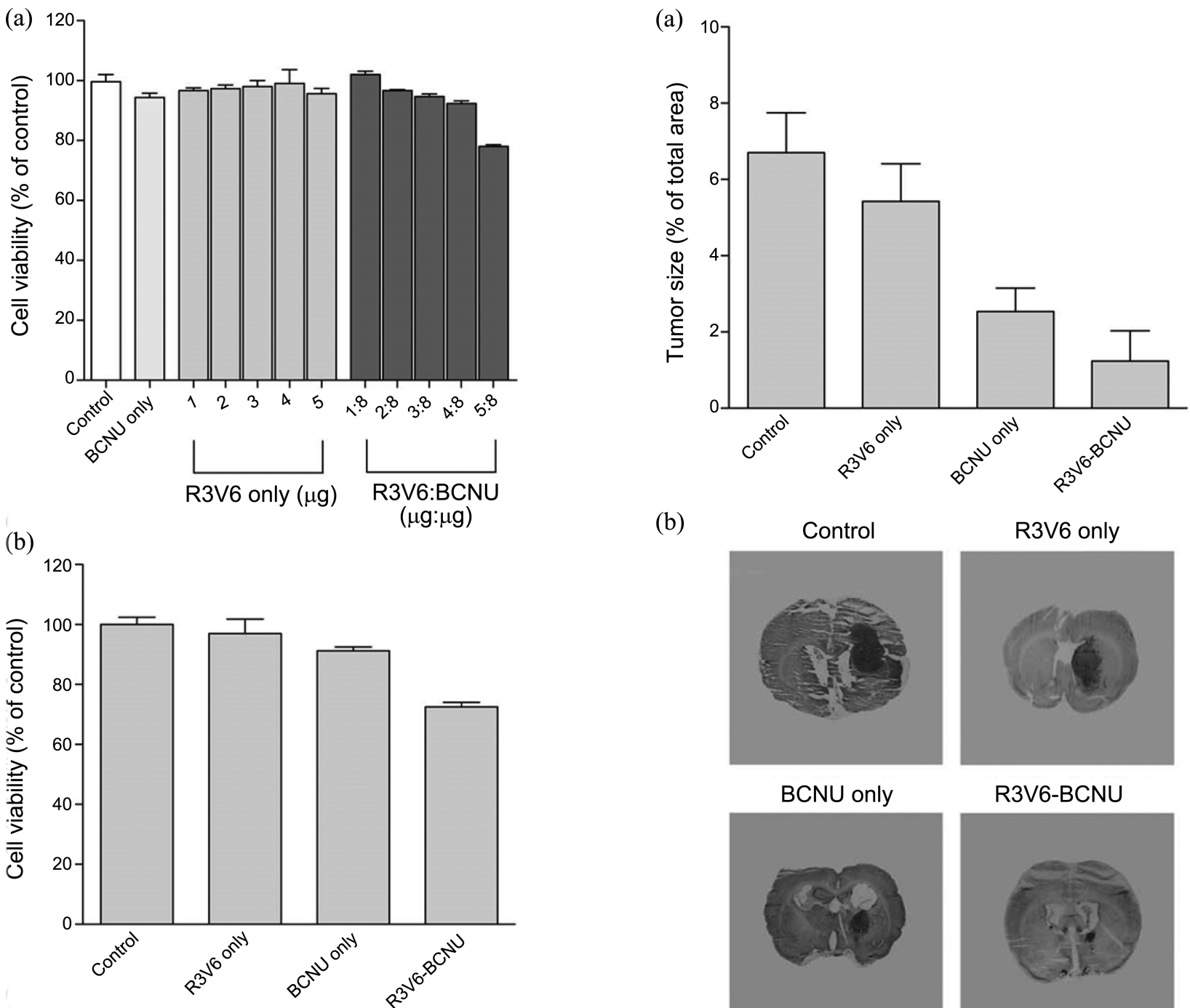

(b)

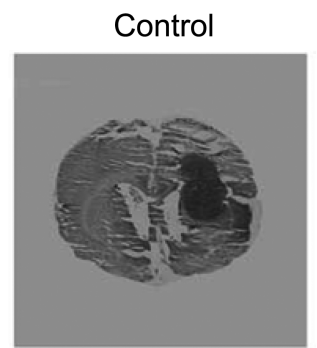

R3V6 only

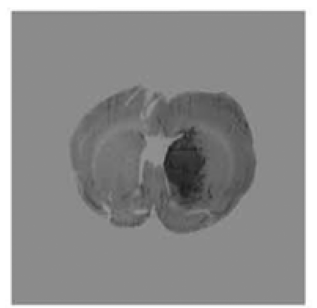

R3V6-BCNU

(c)
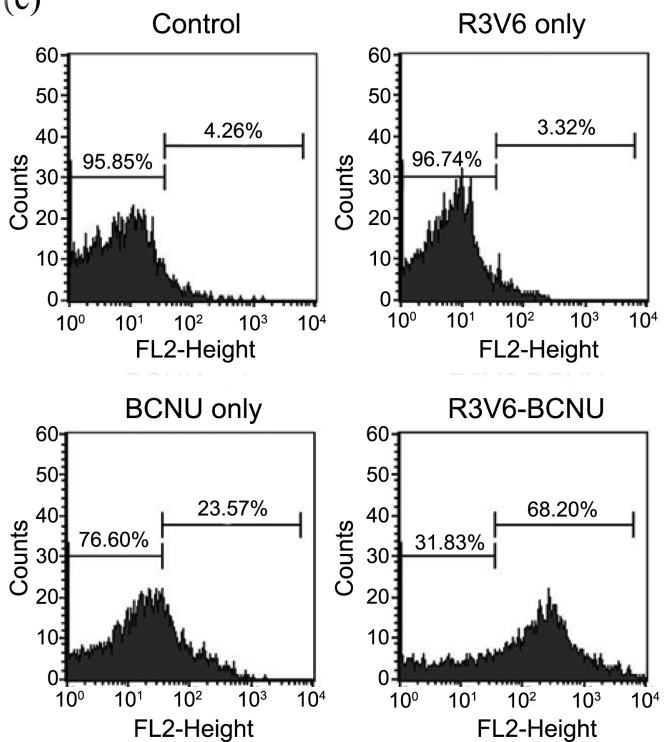

Figure 4. In vitro anti-cancer effect of R3V6-BCNU. The anticancer effects of R3V6-BCNU and BCNU only in C6 glioblastoma cells were measured by MTT assays ( $a$ and $b$ ) and Annexin$\mathrm{V}$ assay (c).
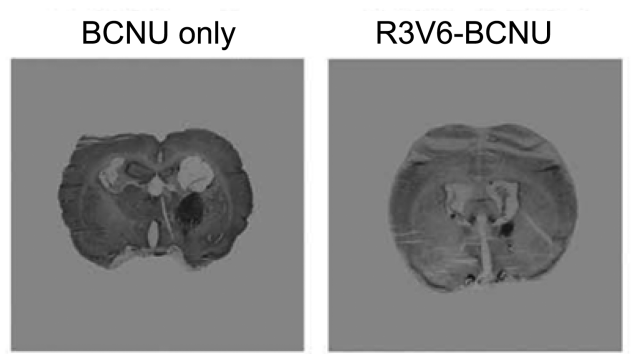

Figure 5. In vivo anti-cancer effect of R3V6-BCNU in an intracranial glioblastoma animal model. (a) Tumor sizes and (b) typical staining results after the injections of saline, R3V6 only, $\mathrm{BCNU}$ only, or R3V6-BCNU. The intracranial glioblastoma animal model was produced as described in the Materials and methods section. R3V6-BCNU, R3V6 only, BCNU only, or saline was injected into the tumor using stereotaxic equipment. One week after the injection, the brains were harvested, and the tumor sizes were measured using Nissl staining.

the tumor size was further decreased by the injection of R3V6-BCNU (Fig. 5). These results confirmed that R3V6$\mathrm{BCNU}$ more efficiently delivered $\mathrm{BCNU}$ into the tumor tissue than did BCNU only.

\section{Discussion}

$\mathrm{BCNU}$ is an anti-cancer drug for glioblastoma. To achieve its anti-cancer effect, BCNU needs to be efficiently internalized into the tumor cells. To engineer a more efficient cellular uptake, BCNU was loaded into R3V6 peptide micelles. In this study, the successful loading of hydrophobic 
drugs into the cores of the R3V6 micelles was confirmed using a dye-quenching assay (Fig. 2). Similarly, the loading of hydrophobic curcumin into the cores of R7L10 micelles has also been confirmed in a previous report. ${ }^{14}$

In the current study, the delivery efficiency of BCNU was evaluated for glioblastoma therapy in an intracranial animal model. The in vitro and in vivo results suggest that R3V6$\mathrm{BCNU}$ increases the anti-cancer efficiency of BCNU. This enhanced anti-cancer effect may be due to the enhanced delivery of BCNU into the cells. In particular, the positive charges of arginines in the peptide micelles may facilitate the interaction between the micelles and the negativelycharged cellular membrane. This interaction between the micelles and the membranes may increase the level of endocytosis. Indeed, similar results were obtained with curcuminloaded R7L10 peptide micelles. The curcumin-loaded R7L10 peptide micelles increased the intracellular delivery of curcumin compared with the administration of curcumin only. Thus, peptide micelles with stretches of arginine stretch and hydrophobic amino acids may be useful for the delivery of hydrophobic drugs into tumor cells.

Another advantage of R3V6 is its low cytotoxicity. Although R3V6-BCNU was toxic to C6 glioblastoma cells, R3V6 only did not induce toxicity to cells (Fig. 4). This indicates that the high toxicity of R3V6-BCNU is not due to the toxicity of R3V6 but to the enhanced delivery of BCNU. The Annexin-V assay also confirmed that R3V6 did not induce apoptosis in C6 glioblastoma cells. The R3V6 peptide micelles were composed of short amphiphilic peptides. Due to low charge-density, the peptide micelles did not induce cytotoxicity.

Loading hydrophobic drugs into the cores of micelles has additional positive effects. R3V6-BCNU was previously prepared and evaluated as a carrier of siRNA and plasmid DNA. ${ }^{10}$ Previous reports have shown that R3V6-BCNU had an improved delivery efficiency of siRNA and plasmid DNA compared with that of R3V6. ${ }^{10,11}$ The improved delivery efficiency of plasmid DNA and siRNA may be due to the stabilization of the micelle structure by provision of stronger hydrophobic cores such as that in BCNU.

Solid tumors have hypoxic regions in their cores and tumor stem cells are usually found within hypoxic regions. ${ }^{3}$ However, due to the lack of blood capillaries the free diffusion of anticancer drug into the core of the tumor is not efficient. This suggests that drug carriers such as R3V6 may be useful to treat the tumor stem cells in the solid tumors.

In conclusion, R3V6 efficiently delivered BCNU into the cancer cells in an intracranial glioblastoma animal model. In addition, R3V6 did not induce cytotoxicity to the cells. Therefore, R3V6 may be a useful carrier of BCNU for glioblastoma therapy.

Acknowledgments. This work was supported by the International Exchange Program for University Researchers 013-2011-1-E00060, National Research Foundation of Korea, Korea.

\section{References}

1. Karpati, G.; Li, H.; Nalbantoglu, J. Cur. Opin. Mol. Therapeut. 1999, 1, 545-552.

2. Louis, D. N. Ann. Rev. Pathol. 2006, 1, 97-117.

3. Kahlert, U. D.; Maciaczyk, D.; Dai, F.; Claus, R.; Firat, E.; Doostkam, S.; Bogiel, T.; Carro, M. S.; Dobrossy, M.; HeroldMende, C.; Niedermann, G.; Prinz, M.; Nikkhah, G.; Maciaczyk, J. J. Neuropathol. Exp. Neurol. 2012, 71, 1086-1099.

4. Amberger-Murphy, V. Current Cancer Drug Targets 2009, 9, 381390.

5. Haar, C. P.; Hebbar, P.; Wallace, G. C. T.; Das, A.; Vandergrift, W. A., III; Smith, J. A.; Giglio, P.; Patel, S. J.; Ray, S. K.; Banik, N. L. Neurochem. Res. 2012, 37, 1192-1200.

6. Salvati, M.; D'Elia, A.; Frati, A.; Brogna, C.; Santoro, A.; Delfini, R. J. Neurosurg. Sci. 2011, 55, 1-6.

7. Perry, J.; Chambers, A.; Spithoff, K.; Laperriere, N. Curr. Oncol. 2007, 14, 189-194.

8. Lee, J.; Hyun, H.; Kim, J.; Ryu, J. H.; Kim, H. A.; Park, J. H.; Lee, M. J. Control. Release 2012, 158, 131-138.

9. Hyun, H.; Lee, J.; Hwang do, W.; Kim, S.; Hyun, D. K.; Choi, J. S.; Lee, J. K.; Lee, M. Biomaterials 2011, 32, 306-315.

10. Yi, N.; Oh, B.; Kim, H. A.; Lee, M. J. Drug Target. 2014, 22, 156164.

11. Park, J. H.; Kim, H. A.; Cho, S. H.; Lee, M. J. Cell Biochem. 2012, 113, 1645-1653.

12. Sheihet, L.; Dubin, R. A.; Devore, D.; Kohn, J. Biomacromolecules 2005, 6, 2726-2731.

13. Yi, Y. H.; Guo, W. C.; Sun, W. W.; Su, T.; Lin, H.; Chen, S. Q.; Deng, W. Y.; Zhou, W.; Liao, W. P. Biologics: Targets \& Therapy 2008, 2, 339-344.

14. Park, J. H.; Kim, H. A.; Park, J. H.; Lee, M. Biomaterials 2012 , 33, 6542-6550. 